\title{
Improved White Matter Cerebrovascular Reactivity after Revascularization in Patients with Steno-Occlusive Disease
}

\author{
(D). McKetton, (D). Venkatraghavan, (D). Rosen, DD.M. Mandell, DK. Sam, Do. Sobczyk, DJ. Poublanc, DE. Gray, (D)A. Crawley,
} (D). Duffin, DJ.A. Fisher, and (DD.J. Mikulis

\begin{abstract}
BACKGROUND AND PURPOSE: One feature that patients with steno-occlusive cerebrovascular disease have in common is the presence of white matter (WM) lesions on MRI. The purpose of this study was to evaluate the effect of direct surgical revascularization on impaired WM cerebrovascular reactivity in patients with steno-occlusive disease.
\end{abstract}

MATERIALS AND METHODS: We recruited 35 patients with steno-occlusive disease, Moyamoya disease $(n=24)$, Moyamoya syndrome $(n=3)$, atherosclerosis $(n=6)$, vasculitis $(n=1)$, and idiopathic stenosis $(n=1)$, who underwent unilateral brain revascularization using a direct superficial temporal artery-to-MCA bypass (19 women; mean age, $45.8 \pm 16.5$ years). WM cerebrovascular reactivity was measured preoperatively and postoperatively using blood oxygen level-dependent (BOLD) MR imaging during iso-oxic hypercapnic changes in end-tidal carbon dioxide and was expressed as $\% \Delta \mathrm{BOLD}$ MR signal intensity per millimeter end-tidal partial pressure of $\mathrm{CO}_{2}$.

RESULTS: WM cerebrovascular reactivity significantly improved after direct unilateral superficial temporal artery-to-middle cerebral artery (STA-MCA) bypass in the revascularized hemisphere in the MCA territory (mean \pm SD, $-0.0005 \pm 0.053$ to $0.053 \pm 0.046$ $\% \mathrm{BOLD} / \mathrm{mm} \mathrm{Hg} ; P<.0001$ ) and in the anterior cerebral artery territory (mean, $0.0015 \pm 0.059$ to $0.021 \pm 0.052 \% \mathrm{BOLD} / \mathrm{mm} \mathrm{Hg} ; P=.005$ ). There was no difference in WM cerebrovascular reactivity in the ipsilateral posterior cerebral artery territory nor in the vascular territories of the nonrevascularized hemisphere $(P<.05)$.

CONCLUSIONS: Cerebral revascularization surgery is an effective treatment for reversing preoperative cerebrovascular reactivity deficits in WM. In addition, direct-STA-MCA bypass may prevent recurrence of preoperative symptoms.

ABBREVIATIONS: $\mathrm{ACA}=$ anterior cerebral artery; $\mathrm{BOLD}=$ blood oxygen level-dependent; $\mathrm{CVR}=$ cerebrovascular reactivity; $\mathrm{MMD}=\mathrm{Moyamoya}$ disease; $\mathrm{PCA}=$ posterior cerebral artery; $\mathrm{P}_{\mathrm{ET}} \mathrm{CO}_{2}=$ end-tidal partial pressure of $\mathrm{CO}_{2} ; \mathrm{STA}=$ superficial temporal artery

T he superficial temporal artery (STA)-middle cerebral artery (MCA) bypass is a surgical revascularization technique often used to bypass upstream in symptomatic steno-occlusive disease. Cerebrovascular reactivity (CVR) is defined as the change in cerebral blood flow $(\mathrm{CBF})$ in response to a vasoactive stimulus, and reflects the ability of the cerebral vasculature to augment $\mathrm{CBF}$

Received July 17, 2018; accepted after revision October 8.

From the Division of Neuroradiology, Joint Department of Medical Imaging (L.M., C.R., D.M.M., K.S., O.S., J.P., E.G., A.C., D.J.M.), Department of Anesthesia and Pain Management (L.V., J.A.F.), University Health Network, Toronto, Ontario, Canada; Russell H. Morgan Department of Radiology and Radiological Science (K.S.), John Hopkins School of Medicine, Baltimore, Maryland; and Department of Physiology (J.D., J.A.F.) and Institute of Medical Sciences (J.D., J.A.F., D.J.M.), University of Toronto, Toronto, Ontario, Canada.

Please address correspondence to David Mikulis, MD, Toronto Western Hospital, Joint Department of Medical Imaging, McLaughlin Pavilion, 3rd Floor, Room 431, 399 Bathurst St, Toronto, ON M5T 2S8, Canada; e-mail: david.mikulis@uhn.ca

= Indicates article with supplemental on-line table.

Indicates article with supplemental on-line photo.

http://dx.doi.org/10.3174/ajnr.A5912 when cerebral perfusion pressure is reduced (pressure autoregulation $)^{1}$ and when neural activity is increased (neurovascular coupling). ${ }^{2}$ Surgical revascularization can improve CVR and reverse the paradoxic reduction in regional blood known as "steal physiology" in the ipsilateral and often in contralateral gray matter (GM) hemispheres, ${ }^{3,4}$ reverse cortical thinning, ${ }^{5}$ improve neurocognitive function, ${ }^{6}$ and reduce further ischemic events. ${ }^{7}$

White matter (WM) may also be adversely affected by stenoocclusive disease. Chronic hypoperfusion can result in ischemic injury, leading to axonal degeneration and glial proliferation. ${ }^{8}$ This effect can result in WM lesions or WM hyperintensities on T2-weighted imaging or FLAIR imaging. WM lesions signify a progressive cerebral small-vessel disease that is often observed in patients with Moyamoya disease (MMD), patients with stroke, and in aging. Age-related reductions in CVR and perfusion have been found in WM lesions, ${ }^{9}$ and WM abnormalities have been associated with reductions in cognitive function. ${ }^{10}$ In addition, there has been a link among impaired WM CVR, cognitive decline 
in Alzheimer disease, ${ }^{11}$ and cognitive dysfunction and hemodynamic impairment due to steno-occlusive disease. ${ }^{12}$ A previous study reported that WM lesions became reversible by revascularization surgery in MMD partly due to regional CBF increase. ${ }^{13}$ Thus, revascularization surgery may help improve WM CVR that could lead to the conservation of or improvement in cognitive function.

The purpose of this retrospective study was to determine the effects of revascularization on WM CVR in patients with intracranial steno-occlusive disease. We identified 35 patients who fit our selection criteria. We measured WM CVR before and after surgical revascularization in the intervened and nonintervened hemispheres to investigate whether regaining CBF would improve WM CVR.

\section{MATERIALS AND METHODS \\ Patients}

The study was approved by the research ethics board of the University Health Network, and all patients signed informed consent. The patients included in this study were selected from a prospectively maintained data base of patients who underwent CVR blood oxygen level-dependent (BOLD) MR imaging testing as part of a series of research ethics board-approved studies at Toronto Western Hospital. The inclusion criteria for this study were the following: 1) patients with intracranial steno-occlusive disease who had a unilateral direct extracranial-intracranial bypass of the superficial temporal artery to MCA (STA-MCA); 2) the presence of steal physiology on the prerevascularization CVR map; 3 ) an available preoperative CVR study and at least 1 postoperative CVR study at 3 months or later postsurgery; 4) both preoperative and postoperative CVR studies performed with the same $\mathrm{CO}_{2}$ stimulus protocol and acquisition parameters; and 5) all patient CVR data being sufficiently free of artifacts to be comparable, as determined by a neuroradiologist experienced in BOLD MR imaging CVR assessment (D.J.M.). Thirty-five patients were selected on the basis of inclusion criteria in this study (19 women; mean age at time of bypass, $45.8 \pm 16.5$ years). Pathology included MMD $(n=24)$, Moyamoya syndrome $(n=3)$, atherosclerosis $(n=6)$, vasculitis (right ICA stenosis) $(n=1)$, and idiopathic stenosis $(n=1)$. Further details are shown in the On-line Table.

\section{Imaging}

MR imaging was performed on a 3T system (Signa HDx platform; GE Healthcare, Milwaukee, Wisconsin) using an 8-channel phased array head coil. For each patient, an anatomic T1weighted 3D spoiled gradient-echo sequence was acquired with the following parameters that varied from before to after 2009: voxel size $=0.781 \times 0.781 \times 2.2 \mathrm{~mm} / 0.85 \times 0.85 \times 1 \mathrm{~mm} ; \mathrm{TR}=$ $7.88 \mathrm{~ms} ; \mathrm{TE}=3 \mathrm{~ms} ;$ matrix size $=256 \times 256 ; 60 / 146$ slices; FOV $=22 \times 22 \mathrm{~cm}$; flip angle $=12^{\circ}$. Each patient had the same pre- and postoperative BOLD MR imaging parameters acquired. As end-tidal partial pressure of $\mathrm{CO}_{2}\left(\mathrm{P}_{\mathrm{ET}} \mathrm{CO}_{2}\right)$ was manipulated, BOLD MR imaging data were acquired using a $\mathrm{T} 2{ }^{*}$-weighted single-shot spiral gradient-echo sequence with an echo-planar readout. Ten out of 35 patients were scanned before 2010 and had the same acquisition protocol for both pre- and postbypass CVRs, and 25 patients were scanned after 2010 and had a different ac- quisition protocol for their pre- and postbypass CVR BOLD scans. The only differences between protocols included the reduction of a $2-\mathrm{mm}$ gap, lowering the flip angle by $5^{\circ}$, and increasing the slices from 20 to 30 . Therefore, the BOLD parameters that varied from before 2010 to after 2010 were the following: voxel size $=3.75 \times 3.75 \times 5 \mathrm{~mm}, 2-\mathrm{mm}$ gap $/$ no gap, $\mathrm{TR}=2$ seconds, $\mathrm{TE}=30 \mathrm{~ms}$, matrix size $=64 \times 64,20 / 30$ slices, $\mathrm{FOV}=24 \times 24$ $\mathrm{cm}$, flip angle $=90^{\circ} / 85^{\circ}$.

\section{Vasodilatory Stimulus}

The control of $\mathrm{P}_{\mathrm{ET}} \mathrm{CO}_{2}$ and the end-tidal pressure of $\mathrm{O}_{2}$ were achieved during MR imaging using an automated gas blender that regulates gas composition and flow to a sequential gas-delivery breathing circuit (RespirAct ${ }^{\mathrm{TM}}$; Thornhill Medical, Toronto, Ontario, Canada) according to the methods previously described. ${ }^{14}$ The automated gas blender was connected to a soft plastic mask sealed to the face using transparent dressing film (Tegaderm Film, 1626W; 3M Health Care, St. Paul, Minnesota). Before and after 2013, the patients underwent either a 2-square wave (step) protocol or 1-square wave followed by a ramp (step and a ramp) protocol for manipulating $\mathrm{P}_{\mathrm{ET}} \mathrm{CO}_{2}$ (from resting partial pressure of $\mathrm{CO}_{2}$ to $10 \mathrm{~mm} \mathrm{Hg}$ above resting) as previously detailed. ${ }^{3,15}$ Both $\mathrm{P}_{\mathrm{ET}} \mathrm{CO}_{2}$ and end-tidal pressure of $\mathrm{O}_{2}$ values were selected from the raw partial pressure of carbon dioxide and partial pressure of $\mathrm{O}_{2}$ tracings and were confirmed post hoc by visual inspection and corrected if needed by a custom-written program (LabVIEW; National Instruments, Austin, Texas). Of the 35 patients studied, 2 patients (P14 and P21) had their first CVR fail due to excessive motion and had their CVR scan restarted with no motion artifacts. This scan was used for analysis. One patient (P11) had the initial postoperative CVR scan fail due to technical issues with the scanner freezing, causing patient distress with the emergency button pressed. The patient returned for their follow-up the following month.

\section{Data and Statistical Analyses}

$\mathrm{MR}$ imaging and $\mathrm{P}_{\mathrm{ET}} \mathrm{CO}_{2}$ data were imported to an independent workstation and preprocessed using AFNI software (http:// afni.nimh.nih.gov/afni), ${ }^{16}$ SPM8 (http://www.fil.ion.ucl.ac.uk/ spm/software/spm12), and Matlab R2015a (MathWorks, Natick, Massachusetts). $\mathrm{P}_{\mathrm{ET}} \mathrm{CO}_{2}$ data were time-shifted to the point of maximum correlation between the rapid changes in $\mathrm{P}_{\mathrm{ET}} \mathrm{CO}_{2}$ and BOLD signal. CVR was calculated as the slope of the line of best fit between the percentage change in BOLD signal versus $\mathrm{P}_{\mathrm{ET}} \mathrm{CO}_{2}$. CVR was calculated on a voxel-by-voxel basis from the slope of a linear least-squares fit of the BOLD signal data series to the $\mathrm{P}_{\mathrm{ET}} \mathrm{CO}_{2}$ values and was expressed as the percentage change in BOLD signal per change in $\mathrm{P}_{\mathrm{ET}} \mathrm{CO}_{2}(\% / \mathrm{mm} \mathrm{Hg})$. The magnitude of the slope in each voxel was color-coded from a color spectrum in which yellow to red denoted a small-to-large positive slope relating to an increase in $\mathrm{CBF}$, and light to dark blue denoted a small-to-large negative slope relating to reversal of CBF (ie, paradoxic vascular steal physiology) (Fig 1).

Anatomic T1-weighted images were segmented into cerebrospinal fluid (CSF), GM and WM. CVR masks were generated containing only WM, and were subsequently transformed into Montreal Neurological Institute space. Unihemispheric WM 


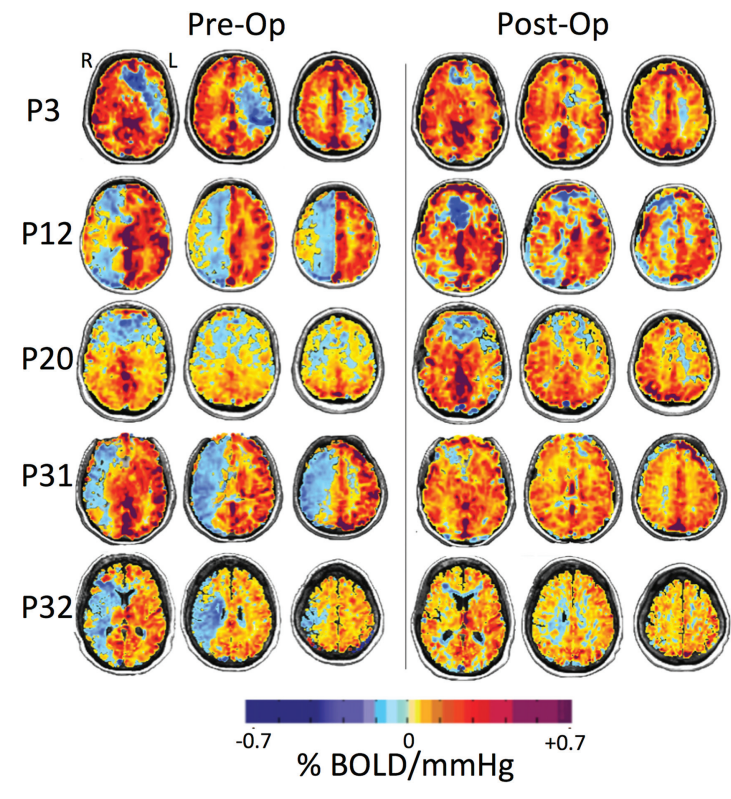

FIG 1. BOLD MR imaging CVR maps in standard space overlaid on an anatomic T1-weighted image of 5 representative patients (P3, P12, P20, $\mathrm{P} 31$, and P32) who underwent a left (P3) and right (P12, P20, P31, and P32) STA-MCA bypass. The first column shows CVR maps before bypass, and the second column shows CVR maps within a 1-year follow-up after revascularization surgery. CVR units are \% $\triangle B O L D$ MR signal intensity per millimeter mercury $\Delta \mathrm{P}_{\mathrm{ET}} \mathrm{CO}_{2}$. Areas shown in blue demonstrate decreased and paradoxic (negative) CVR as shown in P3 in the left hemisphere and P12, P20, P31, and P32 in the right hemisphere (left column). The resulting CVR maps visually demonstrate improvement (reduction of steal) postbypass (right column)

probability maps were thresholded at $70 \%$ probability and were used as a template for calculating CVR in each hemisphere. These were transformed into Montreal Neurological Institute space using SPM8. Unihemispheric statistical parametric mapping GM probability maps were thresholded at 70\% in AFNI and served as a template for calculating hemispheric CVR for each participant. Each major arterial vascular territory comprising the MCA, posterior cerebral artery (PCA), and anterior cerebral artery (ACA) was identified by 2 neuroradiologists (D.J.M. and D.M.M.) and manually traced, creating ROIs. A Wilcoxon matched-pairs signed rank test (2-sided, $\alpha=.05$ ) was used for statistical analysis comparing the prerevascularization and postrevascularization WM CVR values (Fig 2). Results were considered significant after accounting for multiple comparisons by a Bonferroni correction if the per-comparison $P$ value $<.05 /(3$ comparisons) was .017 . Additional analyses on GM CVR values pre- and postrevascularization are reported in the On-line Figure.

\section{RESULTS}

The mean duration between STA-MCA bypass surgery and the postoperative CVR study was 8.3 months (median, 5 months). Each patient's STA-MCA bypass was patent. There was no correlation between follow-up time and WM CVR changes in the revascularized MCA $(r=-0.14, P=.42)$, PCA $(r=-0.12, P=$ $.47)$, and ACA ( $r=0.012, P=.94)$ territory. The mean time between preoperative and postoperative CVR studies was 12.6 months (median, 8 months) (On-line Table).

WM CVR significantly improved after direct unilateral STA-

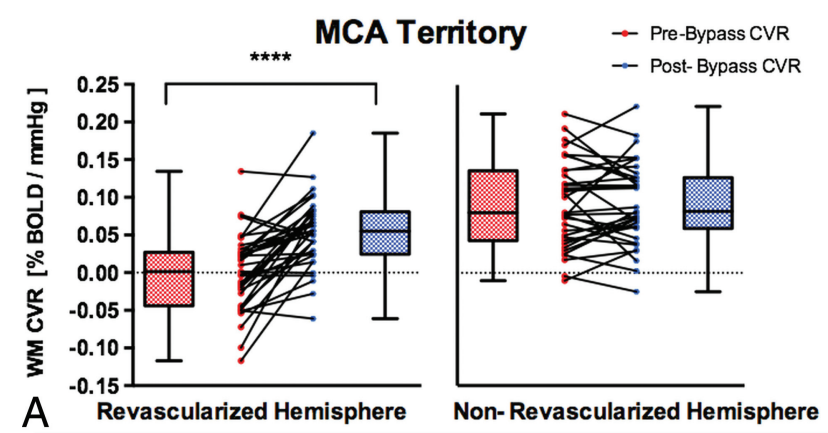

ACA Territory

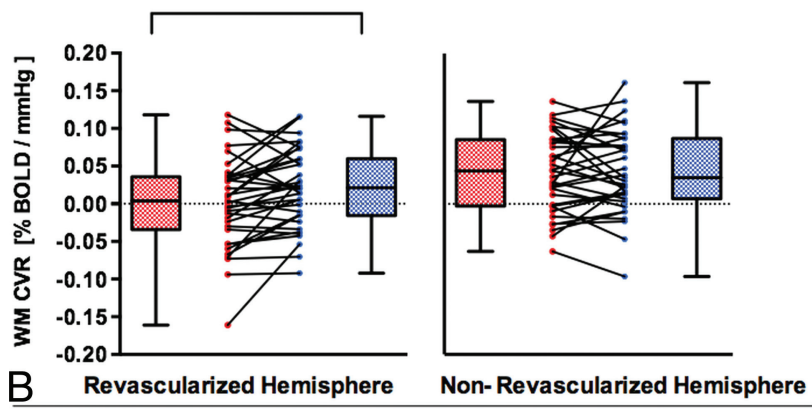

PCA Territory

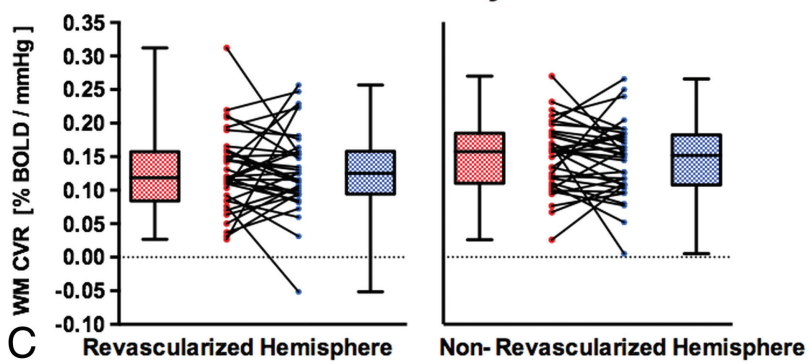

FIG 2. Comparisons of pre- and post-unilateral STA-MCA bypass on white matter CVR in the ipsilateral revascularized and contralateral nonrevascularized hemispheres in patients with MMD, Moyamoya syndrome, and steno-occlusive and atherosclerotic disease. The white matter CVR in revascularized and nonrevascularized hemispheres is shown in different vascular territories: MCA $(A)$, ACA $(B)$, and PCA $(C)$. The box denotes the interquartile range (25\%-75\%), the horizontal line in the box denotes the median, and the whiskers denote the minimum and maximum values. Double asterisks indicate $P<.01 ; 4$ asterisks, $P<.0001$.

MCA bypass in the revascularized hemisphere in the MCA territory (mean $\pm \mathrm{SD},-0.0005 \pm 0.053$ to $0.053 \pm 0.046$ $\% \mathrm{BOLD} / \mathrm{mm} \mathrm{Hg} ; P<.0001)$ and in the ACA territory $(0.0015 \pm$ 0.059 to $0.021 \pm 0.052 \% \mathrm{BOLD} / \mathrm{mm} \mathrm{Hg} ; P=.0053)$. There was no difference in WM CVR after bypass in the ipsilateral PCA territory $(0.128 \pm 0.059$ to $0.13 \pm 0.062 \% \mathrm{BOLD} / \mathrm{mm} \mathrm{Hg} ; P=$ $.98)$ and in the nonrevascularized MCA (mean $\pm \mathrm{SD}, 0.091 \pm$ 0.057 to $0.092 \pm 0.055 \% \mathrm{BOLD} / \mathrm{mm} \mathrm{Hg} ; P=.74), \mathrm{ACA}(0.041 \pm$ 0.052 to $0.043 \pm 0.055 \% \mathrm{BOLD} / \mathrm{mm} \mathrm{Hg} P=.87$ ), or PCA $(0.149 \pm 0.051$ to $0.145 \pm 0.054 \% \mathrm{BOLD} / \mathrm{mm} \mathrm{Hg} ; P=.62)$ territory (Fig 2).

GM CVR significantly improved after direct unilateral STAMCA bypass in the revascularized hemisphere in the MCA territory (mean $\pm \mathrm{SD}, 0.034 \pm 0.07$ to $0.11 \pm 0.059 \% \mathrm{BOLD} / \mathrm{mm} \mathrm{Hg}$; $P<.0001)$. There was no difference in GM CVR after bypass in 
the ipsilateral ACA territory $(0.08 \pm 0.10$ to $0.09 \pm 0.08$ $\% \mathrm{BOLD} / \mathrm{mm} \mathrm{Hg} ; P=.21)$, PCA territory $(0.25 \pm 0.1$ to $0.23 \pm$ $0.1 \% \mathrm{BOLD} / \mathrm{mm} \mathrm{Hg} ; P=.23)$, or in the nonrevascularized MCA $(0.17 \pm 0.1$ to $0.16 \pm 0.08 \% \mathrm{BOLD} / \mathrm{mm} \mathrm{Hg} ; P=.1), \mathrm{ACA}(0.26 \pm$ 0.09 to $0.25 \pm 0.09 \% \mathrm{BOLD} / \mathrm{mm} \mathrm{Hg} ; P=.12)$, or PCA $(0.25 \pm$ 0.08 to $0.25 \pm 0.1 \% \mathrm{BOLD} / \mathrm{mm} \mathrm{Hg} ; P=.09$ ) territory (On-line Figure).

\section{DISCUSSION}

This study shows that the ipsilateral WM CVR improved after unilateral revascularization of the affected hemisphere with a $>10$ time increase in the MCA and ACA territories. In addition, there were some negative changes postbypass in the nonrevascularized hemisphere. For example, of 35 patients, 18 patients had a negative change ranging from a $0.18 \%-10.1 \%$ decrease in CVR postbypass in the nonrevascularized MCA territory (Fig 2A). This could be due to the disease progression affecting the contralateral side and because the WM CVR signal is noisier (ie, the range of $0 \%-10 \%$ pre- and postoperative differences cannot be detected with confidence). Previous studies have shown that revascularization surgery improves cortical GM CVR and reduces the risk of acute ischemia. ${ }^{3,4}$ The importance of our findings suggests that WM CVR impairment, that is related to WM lesions and associated with chronic ischemia, is also improved with revascularization.

Previous studies reported WM CVR characteristics in healthy controls. Sam et al, ${ }^{3}$ reported the mean WM CVR to be $0.17 \% \pm$ $0.06 \% / \mathrm{mm} \mathrm{Hg}$ in 27 healthy control subjects (age range, 19-71 years). Thomas et $\mathrm{al}^{17}$ found that WM CVR was about 4-8 times lower than GM CVR. There is a paucity of literature pertaining to WM CVR postrevascularization.

Our results are in agreement with those of a previous study that found significant improvement in WM CVR in the intervened MCA hemisphere but not in the nonintervened hemisphere after revascularization surgery. ${ }^{3}$ Additionally, Sam et al, ${ }^{3}$ showed that WM CVR improved bilaterally in both ACA and PCA territories in 20 patients with steno-occlusive disease, whereas our findings showed improvement only in the ipsilateral MCA and ACA territories after revascularization. We suggest that these discrepancies may have resulted from sample size differences, heterogeneity in the pathologies of patients, and variability in revascularization techniques in the form of extracranial-intracranial bypass, carotid endarterectomy, and encephaloduroarteriosynangiosis.

Patients with steno-occlusive disease have an increased risk of cerebral hypoperfusion that could consequently lead to brain atrophy, cognitive impairment, and dementia. ${ }^{12}$ The most frequent presentation of intracranial steno-occlusive diseases such as MMD is transient ischemic attack or ischemic stroke. ${ }^{18}$ In MMD, the risk of recurrent stroke is as high as $10 \%$ per year. ${ }^{19}$ Steal physiology in MMD and other steno-occlusive diseases is associated with a high risk of stroke. ${ }^{20}$ Furthermore, impaired CVR with steal physiology in steno-occlusive disease has been associated with cognitive dysfunction, ${ }^{12}$ ipsilateral cortical thinning, ${ }^{21}$ and increased diffusion in $\mathrm{WM}^{22}$; the latter is likely related to increased water diffusivity due to axonal degeneration. ${ }^{23}$ In patients with atherosclerotic disease, CVR deficits are associated with in- creased risk of future ischemic attacks. ${ }^{24}$ However the clinical efficacy of revascularization surgery in decreasing this risk remains controversial. ${ }^{25}$

As a potential benefit to neurocognition, extracranial-intracranial bypass has previously been reported to significantly improve cognitive function in steno-occlusive disease ${ }^{26,27}$; our study suggests that WM reperfusion may contribute to the improvement. Additionally, revascularization surgery has been shown to improve cognitive function in patients with symptomatic primary carotid stenosis undergoing carotid endarterectomy and carotid artery stent placement ${ }^{28}$ and in patients with ICA stenosis undergoing carotid endarterectomy. ${ }^{29}$ The caveat is that these reported results were variable and were part of nonrandomized studies. However, the Randomized Evaluation of Carotid Occlusion and Neurocognition trial reported no significant difference in 2-year cognitive changes between 16 patients who underwent current best medical therapy and 13 patients who underwent extracranial-intracranial bypass. ${ }^{30}$ In both arms, patients had symptomatic ICA occlusion and increased oxygen extraction fraction on PET. Further studies are needed to assess CVR in patients who underwent extracranial-intracranial bypass compared with patients who underwent medical therapy (ie, consistent use of statins and antihypertensives).

The mechanism of restoration of WM CVR requires further investigation, but the phenomenon has been shown previously. Patients with steno-occlusive disease who had undergone extracranial-intracranial arterial bypass surgery had an increase in their total brain blood supply, particularly the restoration of perfusion in hemodynamically compromised brain tissue. ${ }^{31}$ Revascularization surgery was also previously found to improve cerebral perfusion in MMD in the ipsilateral MCA territory in children $^{32}$ and adults. ${ }^{33}$

The assessment of cerebral hemodynamic insufficiency in steno-occlusive disease has remained a complex issue due to the lack of suitable methods and consistency between study centers. ${ }^{34}$ Various imaging methods are used to evaluate cerebral hemodynamics, including CVR or oxygen extraction fraction using PET, ${ }^{35}$ xenon-enhanced $\mathrm{CT}^{36}$, perfusion-weighted MR imaging, ${ }^{37}$ transcranial Doppler, ${ }^{34}$ and SPECT. ${ }^{38}$ BOLD CVR MR imaging and oxygen extraction fraction using PET are the most commonly used mapping methods. However, PET is costly, not widely available, more invasive, uses ionizing radiation, ${ }^{39}$ and provides no indication of dynamic autoregulation. ${ }^{40}$ Standardized BOLD MR imaging CVR using $\mathrm{CO}_{2}$ as a vasodilatory stimulus is noninvasive, requires no exposure to ionizing radiation (important for use in the pediatric population) or contrast agents, and is a more available method for mapping whole-brain CVR. In addition, a recent study in patients with advanced steno-occlusive disease showed that BOLD MR imaging CVR performed very well against PET CVR using identical $\mathrm{CO}_{2}$ stimuli. ${ }^{41}$ BOLD CVR MR imaging is therefore an accessible and readily applied method for routine clinical use.

CVR can be measured using BOLD MR imaging as a surrogate for cerebral blood flow and hypercapnic changes in the $\mathrm{P}_{\mathrm{ET}} \mathrm{CO}_{2}$ as the vasoactive stimulus. ${ }^{42}$ In healthy individuals, there is a strong positive relationship between $\mathrm{P}_{\mathrm{ET}} \mathrm{CO}_{2}$ and $\mathrm{CBF}$ over a large range of $\mathrm{P}_{\mathrm{ET}} \mathrm{CO}_{2}$. In steno-occlusive disease, patients may exhibit 
downstream regional decreases in CBF in response to hypercapnia. With mild hemodynamic impairment, reductions in CVR result from a diminished increase in blood flow, whereas in more severe cases, steal physiology occurs. Vascular steal arises from the redistribution of blood flow away from any region that has reduced vascular reserve to vascular beds with intact reserve, which results in a greater reduction in flow resistance. ${ }^{15}$ In addition to vascular steal, other mechanisms including cerebrovascular autoregulation and/or the cerebral metabolic rate of $\mathrm{O}_{2}$ up-regulation may contribute to negative BOLD CVR. ${ }^{43}$ Here, an alternative explanation for a negative BOLD signal could be due to an increase in blood volume, particularly if the CBV changes happen in arteries or veins that have low oxygen saturation fractions as found in patients with hypoxia with no change in deoxyhemoglobin concentration in the blood. Additionally, Arteaga et $\mathrm{al}^{43}$ reported negative BOLD in WM with low CBV (0.01-0.02 mL blood/mL parenchyma), which would need very sizeable changes in CBV to explain the magnitude of the CVR BOLD signal decrease. Although their stimulus included hyperoxia (and ours did not), it was proposed that the cerebral metabolic rate of $\mathrm{O}_{2}$ may increase during the hypercapnic and hyperoxia stimulus because hypoxic tissue at baseline may metabolize the additional oxygen provided. ${ }^{43}$ Partial voluming with CSF (because CSF has no CBF) in periventricular tissue may have contributed to paradoxic reduced flow; however, because the patients have larger regions of steal, we can rule this out.

There are a number of approaches used to implement a vasoactive stimulus (eg, breath-holding, administering a constant level of $\mathrm{CO}_{2}$, injecting acetazolamide) that can result in large variations in the stimuli and thereby large variations in measured CVR. ${ }^{44}$ Our study used BOLD MR imaging with precisely repeatable $\mathrm{CO}_{2}$, which minimizes sources of variability and allows detailed mapping of the evolution of CVR changes in patients across time, accounting for voxelwise test-retest variability compared against a control CVR atlas. ${ }^{9}$ This key feature is unique to this study because accurate assessments in CVR changes are challenging to separate from test-retest variability with nonrepeatable and unknown stimuli.

The present study did have limitations. The patient population under study included heterogeneous vascular pathology; nevertheless, each patient underwent standardized unilateral direct STA-MCA bypass surgery. We allowed liberal inclusion criteria with respect to age, medication, and disease process to maintain the generality of the results.

\section{CONCLUSIONS}

Unilateral extracranial-intracranial bypass improves WM CVR in the ipsilateral MCA and ACA territories, indicating improvement in underlying hemodynamic reserve. Prospective studies using quantitative, reproducible vasodilatory stimuli examining postrevascularization cognitive performance and other clinical parameters, including patients who have undergone common medical therapy, are warranted and strongly recommended to shed further light on the efficacy of bypass revascularization.

Disclosures: J.A.F. and J.D. are senior scientists at Thornhill Research, Inc., (TRI), a spin-off company affiliated with the University Health Network (UHN) that developed the RespirAct ${ }^{\mathrm{TM}}$, a non-commercial research tool made available by TMI to research institutions to enable CVR studies. D.J.M. is a shareholder in TRI. O.S. is a part time employee of TRI. The remaining authors have no disclosures and declare that the research was conducted in the absence of any commercial or financial relationships that could be construed as a potential conflict of interest.

\section{REFERENCES}

1. Fantini S, Sassaroli A, Tgavalekos KT, et al. Cerebral blood flow and autoregulation: current measurement techniques and prospects for noninvasive optical methods. Neurophotonics 2016;3:31411 CrossRef Medline

2. Pillai JJ, Mikulis DJ. Cerebrovascular reactivity mapping: an evolving standard for clinical functional imaging. AJNR Am JNeuroradiol 2015;36:7-13 CrossRef Medline

3. Sam K, Poublanc J, Sobczyk O, et al. Assessing the effect of unilateral cerebral revascularisation on the vascular reactivity of the non-intervened hemisphere: a retrospective observational study. $B M J$ Open 2015;5:e006014 CrossRef Medline

4. Mandell DM, Han JS, Poublanc J, et al. Quantitative measurement of cerebrovascular reactivity by blood oxygen level-dependent MR imaging in patients with intracranial stenosis: preoperative cerebrovascular reactivity predicts the effect of extracranialintracranial bypass surgery. AJNR Am J Neuroradiol 2011;32: 721-27 CrossRef Medline

5. Fierstra J, Maclean DB, Fisher JA, et al. Surgical revascularization reverses cerebral cortical thinning in patients with severe cerebrovascular steno-occlusive disease. Stroke 2011;42:1631-37 CrossRef Medline

6. Baek HJ, Chung SY, Park MS, et al. Preliminary study of neurocognitive dysfunction in adult moyamoya disease and improvement after superficial temporal artery-middle cerebral artery bypass. $J$ Korean Neurosurg Soc 2014;56:188-93 CrossRef Medline

7. Mesiwala AH, Sviri G, Fatemi N, et al. Long-term outcome of superficial temporal artery-middle cerebral artery bypass for patients with Moyamoya disease in the US. Neurosurg Focus 2008;24:E15 CrossRef Medline

8. Pantoni L, Garcia JH. The significance of cerebral white matter abnormalities $\mathbf{1 0 0}$ years after Binswanger's report: a review. Stroke 1995;26:1293-301 CrossRef Medline

9. Sobczyk O, Crawley AP, Poublanc J, et al. Identifying significant changes in cerebrovascular reactivity to carbon dioxide. AJNR Am J Neuroradiol 2016;37:818-24 CrossRef Medline

10. Deary IJ, Leaper SA, Murray AD, et al. Cerebral white matter abnormalities and lifetime cognitive change: a 67-year follow-up of the Scottish Mental Survey of 1932. Psychol Aging 2003;18:140-48 CrossRef Medline

11. Silvestrini M, Pasqualetti P, Baruffaldi R, et al. Cerebrovascular reactivity and cognitive decline in patients with Alzheimer disease. Stroke 2006;37:1010-15 CrossRef Medline

12. Balucani C, Viticchi G, Falsetti L, et al. Cerebral hemodynamics and cognitive performance in bilateral asymptomatic carotid stenosis. Neurology 2012;79:1788-95 CrossRef Medline

13. Komatsu K, Mikami T, Noshiro S, et al. Reversibility of white matter hyperintensity by revascularization surgery in Moyamoya disease. J Stroke Cerebrovasc Dis 2016;25:1495-502 CrossRef Medline

14. Slessarev M, Han J, Mardimae A, et al. Prospective targeting and control of end-tidal CO2 and $\mathrm{O} 2$ concentrations. J Physiol 2007;581: 1207-19 CrossRef Medline

15. Sobczyk O, Battisti-Charbonney A, Fierstra J, et al. A conceptual model for $\mathrm{CO} 2$-induced redistribution of cerebral blood flow with experimental confirmation using BOLD MRI. Neuroimage 2014;92: 56-68 CrossRef Medline

16. Cox RW. AFNI: software for analysis and visualization of functional magnetic resonance neuroimages. Comput Biomed Res 1996; 29:162-73 CrossRef Medline

17. Thomas BP, Liu P, Park DC, et al. Cerebrovascular reactivity in the brain white matter: magnitude, temporal characteristics, and age effects. J Cereb Blood Flow Metab 2014;34:242-47 CrossRef Medline 18. Zhao WG, Luo Q, Jia JB, et al. Cerebral hyperperfusion syndrome 
after revascularization surgery in patients with moyamoya disease. Br J Neurosurg 2013;27:321-25 CrossRef Medline

19. Chiu D, Shedden $P$, Bratina $P$, et al. Clinical features of moyamoya disease in the United States. Stroke 1998;29:1347-51 CrossRef Medline

20. Schoof J, Lubahn W, Baeumer M, et al. Impaired cerebral autoregulation distal to carotid stenosis/occlusion is associated with increased risk of stroke at cardiac surgery with cardiopulmonary bypass. J Thorac Cardiovasc Surg 2007;134:690-96 CrossRef Medline

21. Fierstra J, Poublanc J, Han JS, et al. Steal physiology is spatially associated with cortical thinning. J Neurol Neurosurg Psychiatry 2010; 81:290-93 CrossRef Medline

22. Conklin J, Fierstra J, Crawley AP, et al. Impaired cerebrovascular reactivity with steal phenomenon is associated with increased diffusion in white matter of patients with Moyamoya disease. Stroke 2010;41:1610-16 CrossRef Medline

23. Helenius J, Soinne L, Salonen O, et al. Leukoaraiosis, ischemic stroke, and normal white matter on diffusion-weighted MRI. Stroke 2002;33:45-50 CrossRef Medline

24. Silvestrini M, Vernieri F, Pasqualetti P, et al. Impaired cerebral vasoreactivity and risk of stroke in patients with asymptomatic carotid artery stenosis. JAMA 2000;283:2122-27 CrossRef Medline

25. Grubb RL, Powers WJ, Derdeyn CP, et al. The Carotid Occlusion Surgery Study. Neurosurg Focus 2003;14:e9

26. Dong Y, Teoh HL, Chan BP, et al. Changes in cerebral hemodynamic and cognitive parameters after external carotid-internal carotid bypass surgery in patients with severe steno-occlusive disease: a pilot study. J Neurol Sci 2012;322:112-16 CrossRef Medline

27. Nielsen H, Højer-Pedersen E, Gulliksen G, et al. Reversible ischemic neurological deficit and minor strokes before and after EC/IC bypass surgery: a neuropsychological study. Acta Neurol Scand 1986; 73:615-18 CrossRef Medline

28. Lal BK, Younes M, Cruz G, et al. Cognitive changes after surgery vs stenting for carotid artery stenosis. J Vasc Surg 2011;54:691-98 CrossRef Medline

29. Yoshida K, Ogasawara K, Kobayashi M, et al. Improvement and impairment in cognitive function after carotid endarterectomy: comparison of objective and subjective assessments. Neurol Med Chir (Tokyo) 2012;52:154-60 CrossRef Medline

30. Marshall RS, Festa JR, Cheung YK, et al; RECON Investigators. Randomized Evaluation of Carotid Occlusion and Neurocognition (RECON) trial: main results. Neurology 2014;82:744-51 CrossRef Medline

31. Neff KW, Horn P, Dinter D, et al. Extracranial/intracranial arterial bypass surgery improves total brain blood supply in selected symptomatic patients with unilateral internal carotid artery occlusion and insufficient collateralization. Neuroradiology 2004;46:730-37 CrossRef Medline

32. Blauwblomme $\mathrm{T}$, Lemaitre $\mathrm{H}$, Naggara $\mathrm{O}$, et al. Cerebral blood flow improvement after indirect revascularization for pediatric Moyamoya disease: a statistical analysis of arterial spin-labeling MRI. AJNR Am J Neuroradiol 2016;37:706-12 CrossRef Medline
33. Kwon WK, Kwon TH, Park DH, et al. Efficacy of superficial temporal artery-middle cerebral artery bypass in cerebrovascular stenoocclusive diseases: hemodynamics assessed by perfusion computed tomography. Asian J Neurosurg 2017;12:519-24 CrossRef Medline

34. Reinhard M, Schwarzer G, Briel M, et al. Cerebrovascular reactivity predicts stroke in high-grade carotid artery disease. Neurology 2014; 83:1424-31 CrossRef Medline

35. Endo $\mathrm{H}$, Inoue $\mathrm{T}$, Ogasawara $\mathrm{K}$, et al. Quantitative assessment of cerebral hemodynamics using perfusion-weighted MRI in patients with major cerebral artery occlusive disease: comparison with positron emission tomography. Stroke 2006;37:388-92 CrossRef Medline

36. Meyer JS, Shinohara T, Imai A, et al. Imaging local cerebral blood flow by xenon-enhanced computed tomography? Technical optimization procedures. Neuroradiology 1988;30:283-92 CrossRef Medline

37. Zakariaee S, Oghabian M, Firouznia K, et al. Assessment of the agreement between cerebral hemodynamic indices quantified using dynamic susceptibility contrast and dynamic contrast-enhanced perfusion magnetic resonance imaging. J Clin Imaging Sci 2018;8:2 CrossRef Medline

38. Cikrit DF, Dalsing MC, Harting PS, et al. Cerebral vascular reactivity assessed with acetazolamide single photon emission computer tomography scans before and after carotid endarterectomy. Am JSurg 1997;174:193-97 CrossRef Medline

39. Ragan DK, McKinstry R, Benzinger T, et al. Depression of wholebrain oxygen extraction fraction is associated with poor outcome in pediatric traumatic brain injury. Pediatr Res 2012;71: 199-204 CrossRef Medline

40. Derdeyn CP, Videen TO, Yundt KD, et al. Variability of cerebral blood volume and oxygen extraction: stages of cerebral haemodynamic impairment revisited. Brain 2002;125:595-607 CrossRef Medline

41. Fierstra J, van Niftrik C, Warnock G, et al. Staging hemodynamic failure with blood oxygen-level-dependent functional magnetic resonance imaging cerebrovascular reactivity: a comparison versus gold standard (15O-)H2O-positron emission tomography. Stroke 2018;49:621-29 CrossRef Medline

42. Mandell DM, Han JS, Poublanc J, et al. Mapping cerebrovascular reactivity using blood oxygen level-dependent MRI in patients with arterial steno-occlusive disease: comparison with arterial spin labeling MRI. Stroke 2008;39:2021-28 CrossRef Medline

43. Arteaga DF, Strother MK, Faraco CC, et al. The vascular steal phenomenon is an incomplete contributor to negative cerebrovascular reactivity in patients with symptomatic intracranial stenosis. J Cereb Blood Flow Metab 2014;34:1453-62 CrossRef Medline

44. Powers WJ, Clarke WR, Grubb RL, et al. Extracranial-intracranial bypass surgery for stroke prevention in hemodynamic cerebral ischemia: the Carotid Occlusion Surgery Study randomized trial. JAMA 2011;306:1983 CrossRef Medline 